\title{
The past and the future of the Journal of Ethology: a message from the new editor-in-chief
}

\author{
Kensuke Nakata ${ }^{1}$
}

Published online: 28 November 2016

(C) Japan Ethological Society and Springer Japan 2016

The first volume of the Journal of Ethology was published in 1983, in association with the establishment of the Japan Ethological Society. Since then, the journal had published more than 1000 articles. The first volume of the journal, of which Dr. Toshitaka Hidaka was the first chief editor, had only 14 articles, 13 of which were written by Japanese authors. The journal soon had two issues, including 20-30 articles (approximately half of which were Japanese articles) per year under the chief editors, Drs. Takeji Kimura, Eiiti Kasuya, and Yutaka Saito. Under the fifth chief editor, Dr. Kazuki Tsuji, the number of published articles started increasing. In the past decade, the journal has published 40-60 articles in three issues per year under the chief editors, Drs. Keisuke Ueda, Yoshitaka Tsubaki, and Yukio Yasui. While the journal is still the target for Japanese researchers who want to publish their work, it now also publishes a greater number of good articles submitted by scientists throughout the world. We have 39 open access articles, most of which were published in the last 4 years. All this shows that the journal has undergone steady growth during the past 35 years and has risen to international status as the result of the constant efforts of successive editorial teams, valuable comments from reviewers, and submissions of manuscripts which are both of a high level and interesting.

The eighth editor-in-chief, Dr. Yukio Yasui, will complete his term in December 2016. I have served as an associate editor, mainly handling articles on spiders and crustaceans, for 12 years, and will be the ninth editor-inchief from 2017. There will also be some changes to the

Kensuke Nakata

nakatake@kyoto-wu.ac.jp

1 Kyoto Women's University, Kyoto, Japan editorial team: two new associate editors, Dr. Tsunenori Koga of Wakayama University, Japan, and Dr. Zenobia Lewis of the University of Liverpool, UK, will join us. I will no longer work as an associate editor, but keep handling video articles.

The editorial policy remains the same. The journal accepts articles from a wide field of behavior-related studies, such as behavioral ecology, behavioral physiology, biomechanics, psychology, and applied ethology. The methods can also be variable; field, laboratory, observational, experimental and theoretical studies are all welcome. In the last 3 years, articles whose subject species are birds and mammals, including humans, have accounted for half of the papers published. Fishes and insects were the next largest subject taxa. There were also many articles on spiders, crustaceans, lizards and amphibians; of course, we do not limit the range of subject organisms.

Following the journal's conventions, purely descriptive studies can also be accepted, if they have found a truly novel behavior that is of interest not only to specialists on the subject animal but also to ethologists in general, or have a strong theoretical background. We hold in high regard the value of descriptions of novel behavior, and believe that this attitude contributes to the development of research on organismal behavior.

I believe that knowledge of organismal behavior contributes to various fields, including biology, conservation, robotics, education, entertainment and human and animal welfare. Therefore, I hope that the Journal of Ethology also shows the value of behavioral study to researchers outside our discipline and encourages collaboration with them. Fortunately, the study of organismal behavior has an advantage: it easily attracts wide interest from non-ethologists. The use of video is also useful for this purpose, and 
we have already established the use of video articles in the journal.

The future of the journal depends on how many papers of a high standard we can publish. Our enthusiasm for animal behavior is the fundamental driving force for the development of this field. I hope that many researchers will consider our journal and submit their work to us. 